\title{
MicroRNA-98 and microRNA-214 post-transcriptionally regulate enhancer of zeste homolog 2 and inhibit migration and invasion in human esophageal squamous cell carcinoma
}

\author{
Sheng-Dong Huang ${ }^{1 * \dagger}$, Yang Yuan ${ }^{1 \dagger}$, Chong-Wen Zhuang ${ }^{4}$, Bai-Ling Li ${ }^{2}$, De-Jun Gong ${ }^{3}$, Shu-Gang Wang ${ }^{5}$,
} Zhi-Yong Zeng ${ }^{4^{*}}$ and He-Zhong Cheng ${ }^{1,2^{*}}$

\begin{abstract}
Background: The enhancer of zeste homolog 2 (EZH2) was found to be overexpressed and associated with tumor metastasis in esophageal squamous cell carcinoma (ESCC). On the other hand, it was reported that miR-26a, miR-98, miR-101, miR-124, miR-138 and miR-214 could inhibit the expression of EZH2 in some tumors. However, the role of miRNAs in the regulation of EZH2 expression in human ESCC has not been documented. The aim of this study was to determine the role of these miRNAs in the regulation of tumor metastasis via EZH2 overexpression in human ESCC.

Methods and results: The expression of these miRNAs and EZH2 mRNA were examined by qPCR and the expression of EZH2 protein was detected by western blot. The role of these miRNAs in migration and invasion was studied in ESCC cell line (Eca109) transfected with miRNA mimics or cotransfected with miRNA mimics and pcDNA-EZH2 plasmid (without the 3'-UTR of EZH2). Through clinical investigation, we found that miR-98 and miR-214 expression was significantly lower in ESCC tissues than in matched normal tissues, and the expression level of miR-98 and miR-214 was inversely correlated to EZH2 protein expression and the clinical features such as pathological grade, tumor stage and lymph node metastasis in ESCC. In Eca109 cells, overexpression of miR-98 and miR-214 significantly inhibited the migration and invasion of ESCC cells, which was reversed by transfection of $\mathrm{EZH} 2$.
\end{abstract}

Conclusions: These findings suggest that decreased expression of miR-98 and miR-214 might promote metastasis of human ESCC by inducing accumulation of EZH2 protein.

Keywords: MiR-98, MiR-214, EZH2, ESCC, Migration, Invasion

\section{Introduction}

Esophageal squamous cell carcinoma (ESCC) is the second most common cancer in China [1]. The recurrence rate of ESCC is extremely high after surgical treatment and the prognosis is usually poor $[2,3]$.

\footnotetext{
*Correspondence: dr.hezhongCheng@gmail.com; zengzhiyong623@sina. com; dr.hezhongCheng@gmail.com

${ }^{\dagger}$ Equal contributors

${ }^{1}$ Institute of Cardiothoracic Surgery, Changhai Hospital, 168, Changhai Rd. Shanghai, P. R. China

${ }^{2}$ Department of Cardiothoracic Surgery, Changhai Hospital, Shanghai, P.R. China

Full list of author information is available at the end of the article
}

Metastasis is a strong independent prognostic factor for ESCC [4,5]. Therefore, any insight into the mechanisms of ESCC metastasis may provide important clues for the development of clinical diagnostic methods and effective therapeutics [6].

The enhancer of zeste homolog 2 (EZH2, also called histone lysine methyltransferase) is located at chromosome 7q35 and encodes a member of the Polycomb group proteins [7], which regulate gene expression via epigenetic modification of chromatin structure including inducing histone acetylation and methylation $[7,8]$. Previous studies showed that 
EZH2 is overexpressed in a broad range of tumors $[9,10]$. Moreover, increased EZH2 expression could significantly promote tumor cell migration and invasion, and is strongly associated with tumor metastasis and poor clinical prognosis in a variety of human tumors such as breast, prostate, endometrial, gastric, colon, hepatocellular, bladder and oral cancers [1118]. In the case of human ESCC, He et al. [19] and Tzao et al. [20] independently reported that overexpression of EZH2 is associated with tumor metastasis and poor prognosis of the patients. However, the mechanism underlying $\mathrm{EZH} 2$ overexpression in ESCC remains unclear.

In recent years, accumulating data indicate that expression of EZH2 is regulated at the posttranscriptional level by a number of microRNAs (miRNAs). It was reported that miR-26a, miR-98, miR-101, miR124, miR-138 and miR214 inhibit the expression of EZH2 in nasopharyngeal carcinoma, nasopharyngeal carcinoma, glioblastoma, hepatocellular carcinoma, head and neck squamous cell carcinoma, and neuroblastoma, respectively [21-26]. However, the role of miRNAs in the regulation of EZH2 expression in human ESCC has not been documented. Considering that the expression and function of miRNAs may vary in different types of tumors, here we set out to investigate whether these miRNAs (miR-26a, miR-98, miR-101, miR-124, miR-138 and miR214) regulate tumor metastasis via altering EZH2 expression in human ESCC. Through clinical investigation and cellular experiments using ESCC cell line, we demonstrate that decreased expression of miR-98 and miR214 induce accumulation of $\mathrm{EZH} 2$ protein and might thereby promote the metastasis of human ESCC.

\section{Results \\ EZH2 expression is up-regulated at the post-transcriptional level in ESCC specimens}

In the present study, we examined EZH2 mRNA and protein expression in ESCC tissues and matched normal esophageal tissues by qRT-PCR and western blot analysis. EZH2 mRNA appeared to be higher in tumor than in the normal tissue in 60\% (24/40) of specimens, but overall the difference in EZH2 mRNA expression between ESCC tissues and the matched normal tissues was not statistically significant (Figure 1A). On the other hand, the expression level of EZH2 protein was upregulated in tumor in $92.5 \%(37 / 40)$ of specimens with a total average of 2.6 fold increase than in the matched normal tissues (Figure 1B, Additional file 1: Figure S1). These data suggest that EZH2 expression is up-regulated at the posttranscriptional level in human ESCC.
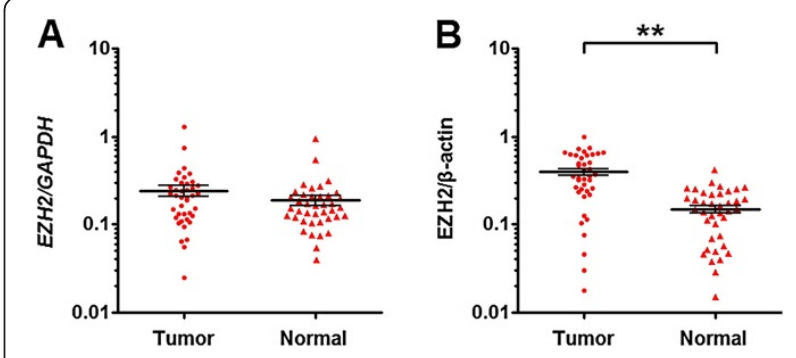

Figure $1 \mathrm{EZH} 2$ protein but not mRNA expression is increased in ESCC specimens as compared to matched normal tissues. (A, B) The expression of EZH2 mRNA and protein in the ESCC specimens and the matched normal tissues was detected by qRT-PCR and western blot and normalized to that of GAPDH and $\beta$-actin, respectively. Results showed that the expression of EZH2 mRNA (C) was comparable between tumor tissue and matched normal tissue, while the expression of EZH2 protein (D) was significantly increased in tumor tissue compared with the matched normal tissue. Data are presented as individual samples $(n=40)$ with the line indicating the mean level; ${ }^{*}, P<0.01$ by paired t test.

\section{MiR-98, miR-101 and miR214 expression is} down-regulated in ESCC specimens

MiR-26a, miR-98, miR-101, miR-124,miR-138 and miR-214 were reported to be decreased in some human tumors and posttranscriptionally regulate the expression of EZH2 [21-26]. In the present study, we first examined the expression levels of MiR-26a, miR-98, miR-101, miR-124, miR138 and miR214 in clinical samples of ESCC and matched normal tissues using qPCR. Expression of miR-98 and miR101 was found to be downregulated in tumor tissues compared with the matched normal tissues in $67.5 \%$ (27/40) of samples. In $77.5 \%(31 / 40)$ of samples, miR-214 was found to be downregulated. In $42.5 \%$ (17/40) of samples, all the three miRNAs were downregulated in tumor versus normal tissues. Figure $2 \mathrm{~A}-\mathrm{C}$ shows the mean expression levels of miR-98, miR-101 and miR-214, which were significantly lower in tumor tissues than in matched normal tissues. On the other hand, expression of miR-138 was significantly higher in tumor tissues than in matched normal tissues (Figure 2D) and the expression levels of miR-26a and miR124 were not significantly different between tumor and matched normal tissues (Figure 2E and F).

We further investigated the relationship of the expression level of miRNAs (miR-98, miR-101 and miR214) with EZH2 expression. It was found that $75 \%$ (30/40), $62.5 \%(25 / 40)$, and $85 \%(34 / 40)$ of samples showed downregulation of miRNAs (i.e.. miR-98, miR-101 and miR214) with upregulation of EZH2 protein in tumor versus normal tissues, respectively. These results suggest that downregulation of these miRNAs might contribute to the accumulation of EZH2 protein in tumors. Moreover, the expression of miR-98 (or miR-214) was inversely correlated with EZH2 protein but not mRNA expression in tumor tissues (Figure 3A-D). On the other hand, there 

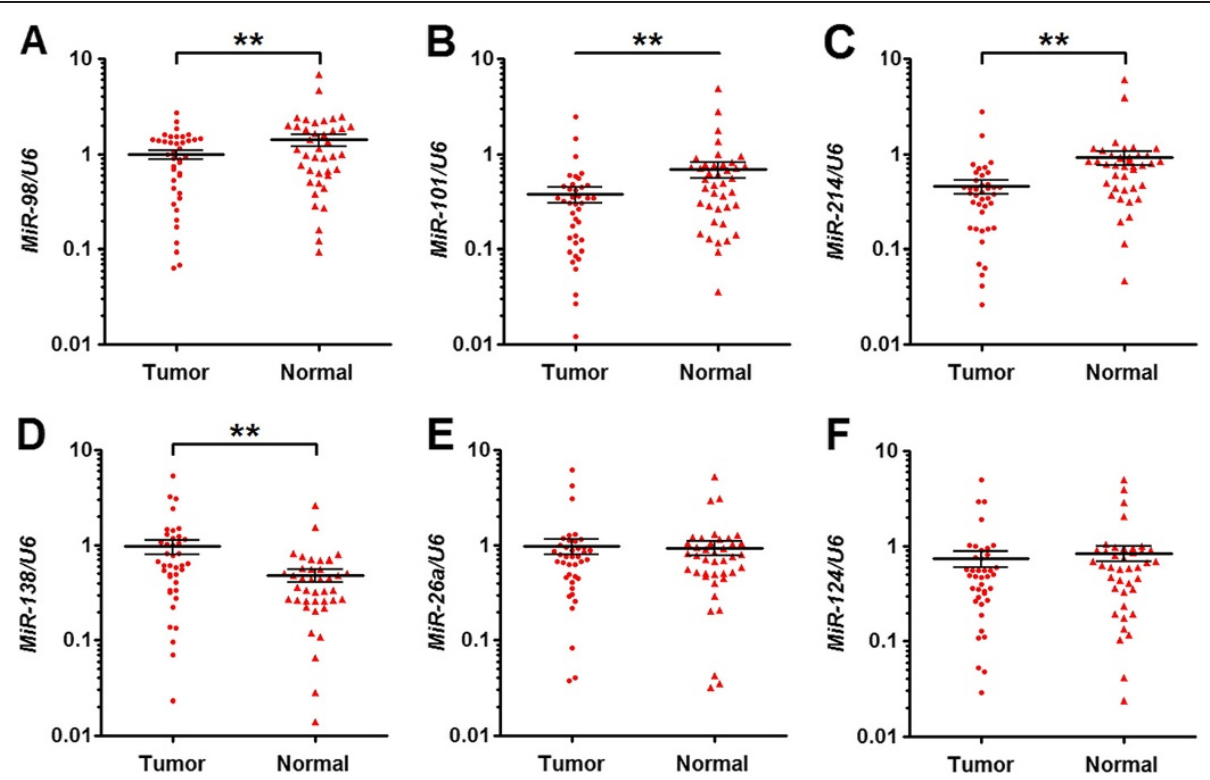

Figure 2 MiR-98, miR-101 and miR-214 expression is decreased in ESCC specimens as compared to matched normal tissues. The expression of miRNAs in the ESCC specimens and the matched normal tissues was detected by qRT-PCR and normalized to that of U6. Results showed that the expression of miR-98 (A), miR-101 (B) and miR-214 (C) were significantly decreased in tumor tissue compared with the matched normal tissue; while that of miR-138 (D) was significantly increased in tumors tissue, and there was no significantly difference in the expression of miR-26a $(\mathbf{E})$ and miR-124 (F) between the two groups. Data are presented as individual samples $(n=40)$ with the line indicating the mean level; **, $P<0.01$ by paired t test.


Figure 3 MiR-98 and miR-214 expression is inversely correlated with that of EZH2 protein. Dot plots represent $\log _{10} \mathrm{EZH} 2$ protein (mRNA) relative expression level against $\log _{10}$ miRNA relative expression level. The lines represent approximated curves. The correlation coefficient ( $r$ ) and the $P$ value indicate the statistical significance of the negative correlation between the $\mathrm{x}$ and $\mathrm{y}$ variables. Results showed that the expression of miR-98 was inversely correlated with EZH2 protein (A) but not mRNA expression (B) in tumor cells. Similarly, the expression of miR-214 was inversely correlated with EZH2 protein (C) but not mRNA expression (D). There was no significant association between the expression of miR-101 and $\mathrm{EZH} 2$ protein $(\mathbf{E})$ and $\mathrm{mRNA}(\mathbf{F})$. 
appeared to be no significant association between the expression of miR-101 and EZH2 mRNA and protein (Figure 3E-F). These data suggest that miR-98 and miR-214 may play an important role in regulating the expression of EZH2 protein in human ESCC.

\section{MiR-98, miR-101 and miR214 are correlated with pathological grade, tumor stage and lymph node metastasis}

We further analyzed the relationship of miR-98, miR-101 and miR214 expression with the clinical features including age, gender, pathological grade, tumor location, tumor stage and lymph node metastasis in ESCC. For each miRNA, patients were divided into two groups, high expression (meaning higher than the mean level) and low expression (meaning lower than the mean level). It was found that the expression of miR-98 (or miR-214) was significantly correlated with pathological grade, tumor stage and lymph node metastasis (Table 1). MiR-101 expression was also significantly correlated with tumor stage and lymph node metastasis (Table 1). These results suggest that the miR-98, miR101 and miR214 might be involved in metastasis of ESCC.

\section{MiR-98, miR-101 and miR-214 posttranscriptionally} down-regulates EZH2 expression in ESCC cell line To determine whether the 3'-UTR of EZH2 mRNA is a functional target of miR-98, miR-101 and miR-214 in ESCC cells, we measured the luciferase activity in cells cotransfected with these miRNAs (or control miRNA) and LucEZH2 plasmid (or Luc-EZH2-mut plasmid) in ESCC cell line (Eca109). qPCR analysis confirmed that Eca109 cells transfected with miRNA mimics (i.e miR-98, miR-101 or miR-214) exhibited significantly higher mature miRNA level than those treated with controls $48 \mathrm{hr}$ posttransfection (Additional file 2: Figure S2). As shown in Figure 4A-C, cells cotransfected with miRNAs (ie, miR-98, miR-101 or miR-214) and Luc-EZH2 plasmid showed a significant decrease of reporter activity in comparison with those cotransfected with control microRNA and Luc-EZH2 plasmid. However, the reporter activity were comparable between cells cotransfected with miRNAs (miR-98, miR-101 ormiR-214) and Luc-EZH2-mut plasmid and cells cotransfected with control microRNA and Luc-EZH2-mut plasmid (Figure 4A-C). We further detected the expression of EZH2 protein and mRNA by western blot and qRT-PCR in Eca109 cells transfected with miRNAs (miR-98, miR-101 or miR-214). Expression of EZH2 protein was found to be significantly decreased in cells transfected with miRNAs (miR-98. miR-101 or miR-214) as compared to cells treated with control microRNA (Figure 4D). However, EZH2 mRNA expression was not significantly different between the 2 groups (Figure 4E). These results indicate that the 3'-UTR of EZH2 mRNA is a functional target of miR-98, miR-101 and miR-214 in ESCC cells.

Table 1 Relationship between the expression of miRNAs (miR-98, miR-101 and miR-214) and clinical features

\begin{tabular}{|c|c|c|c|c|c|c|c|c|c|}
\hline \multirow[t]{2}{*}{ Clinical features } & \multicolumn{3}{|c|}{ MiR-98 } & \multicolumn{3}{|c|}{ MiR-101 } & \multicolumn{3}{|c|}{ MiR-214 } \\
\hline & High & Low & $P$ & High & Low & $P$ & High & Low & $P$ \\
\hline Total & 20 & 20 & & 13 & 27 & & 12 & 28 & \\
\hline \multicolumn{10}{|l|}{ Age (year) } \\
\hline$<60$ & 7 & 9 & 0.519 & 7 & 9 & 0.215 & 4 & 12 & 0.573 \\
\hline$\geq 60$ & 13 & 11 & & 6 & 18 & & 8 & 16 & \\
\hline \multicolumn{10}{|l|}{ Gender } \\
\hline Male & 16 & 12 & 0.168 & 7 & 21 & 0.122 & 8 & 20 & 0.763 \\
\hline Female & 4 & 8 & & 6 & 6 & & 4 & 8 & \\
\hline \multicolumn{10}{|l|}{ Pathological grading } \\
\hline Well & 11 & 2 & 0.004 & 7 & 6 & 0.135 & 8 & 5 & 0.010 \\
\hline Moderately & 6 & 7 & & 3 & 10 & & 2 & 11 & \\
\hline Poorly & 3 & 11 & & 3 & 11 & & 2 & 12 & \\
\hline \multicolumn{10}{|l|}{ Tumor location } \\
\hline Upper1/3-middle1/3 & 15 & 12 & 0.311 & 8 & 19 & 0.576 & 9 & 18 & 0.507 \\
\hline Lower1/3 & 5 & 8 & & 5 & 8 & & 3 & 10 & \\
\hline \multicolumn{10}{|l|}{ Tumor stage } \\
\hline $\mathrm{T} 1 / \mathrm{T} 2$ & 12 & 3 & 0.003 & 9 & 6 & 0.004 & 8 & 7 & 0.013 \\
\hline $\mathrm{T} 3 / \mathrm{T} 4$ & 8 & 17 & & 4 & 21 & & 4 & 21 & \\
\hline \multicolumn{10}{|l|}{ Lymph node metastasis } \\
\hline Negative & 13 & 4 & 0.004 & 9 & 8 & 0.018 & 8 & 9 & 0.043 \\
\hline Positive & 7 & 16 & & 4 & 19 & & 4 & 19 & \\
\hline
\end{tabular}



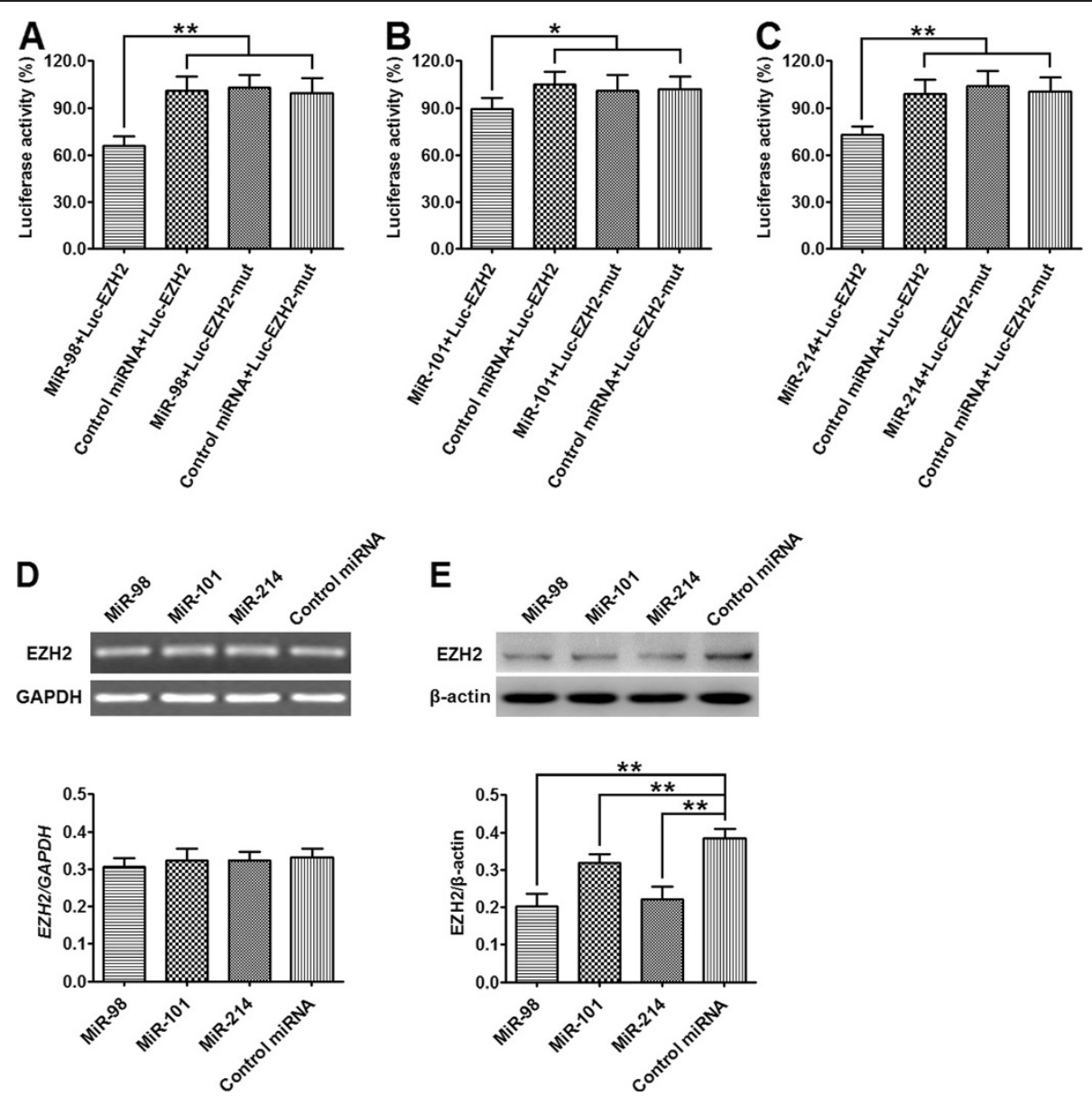

Figure 4 MiR-98, miR-101 and miR-214 posttranscriptionally down-regulates EZH2 expression in ESCC cell line. (A-C) $1 \times 10^{6}$ Eca109 cells were cotransfected with 50 pmol of miRNAs (or control miRNA) and $1 \mu \mathrm{g}$ of Luc-EZH2 (or matched Luc-EZH2-mut) plasmid, respectively. Luciferase reporter assay were performed at $48 \mathrm{hr}$ posttransfection. Results showed that cells transfected with miR-98 + Luc-EZH2 (A), miR-101 Luc-EZH2 (B), miR-214 Luc-EZH2 (C) exhibited a significant decrease of reporter activity in comparison with those cotransfected with control microRNA + Luc-EZH2 plasmid. However, the reporter activity of cells cotransfected with miRNAs and Luc-EZH2-mut plasmid showed no significant difference with that of cells cotransfected with control miRNA and Luc-EZH2-mut plasmid. (D, E) $1 \times 10^{6}$ Eca109 cells were transfected with 50 pmol of miRNAs (or control microRNA), respectively. The expression level of EZH2 mRNA and protein was detected by qPT-PCR an Western Blot at $48 \mathrm{hr}$ posttransfection and normalized to that of GAPDH and $\beta$-actin, respectively. Results showed that the level of EZH2 protein (D) was significantly decreased in cells transfected with miRNAs (including miR-98, miR-101 and miR-214) as compared to the cells transfected with control microRNA, while the expression level of EZH2 mRNA (E) exhibited no significantly difference between cells transfected with miRNAs and those transfected with control microRNA. Data represent mean \pm SEM from 4 independent experiments; ${ }^{*}, P<0.05,{ }^{*}, P<0.01$ by $t$ test.

\section{MiR-98, miR-101 and miR-214 inhibit the migration and invasion of ESCC cell line}

To investigate the role of miR-98, miR-101 and miR-214 in ESCC metastasis, we detected the migrant and invasive capacity of Eca109 cells transfected with miRNA mimics or control miRNA. Through transwell assay, we found that the percentage of cells travelled through the micropore membrane was significantly decreased in cells transfected with miRNAs (miR-98, miR-101 or miR-214) as compared to those cells transfected with control miRNA (Figure 5A). In addition, using matrigel-coated transwell assay, we found that the percentages of cells that invaded through the matrigel in miRNAs (miR-98, miR-101 or miR-214) transfected cells were significantly lower than those in the control groups (Figure $5 \mathrm{~B}$ ). These results indicate that miR-98, miR-101 and miR-214 could inhibit the migration and invasion of ESCC cells.

\section{Overexpression of EZH2 reverses the inhibition of migration and invasion of ESCC cells by miR-98 and miR-214}

To investigate the functional connection between miRNAs (miR-98, miR-101 and miR-214) and EZH2 in the regulation of ESCC metastasis, we further evaluated the migration and invasion capacity of cells cotransfected with these miRNAs and pcDNA-EZH2 (or empty pcDNA) plasmid. Notably, the pcDNA-EZH2 plasmid was designed 

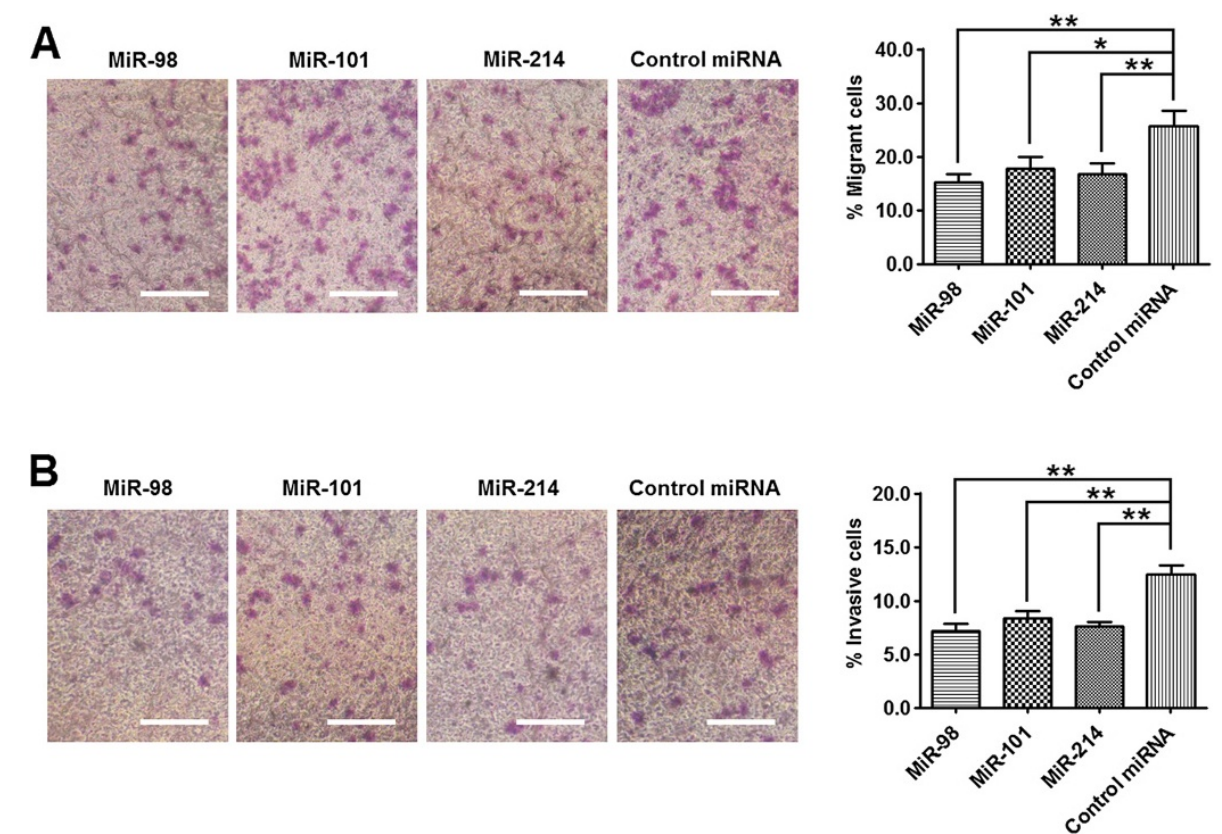

Figure 5 MiR-98, miR-101 and miR-214 inhibits the migration and invasion of ESCC cells. Eca109 cells were transfected with 50 pmol of miRNAs (or control microRNA). Migration and invasion of cells were analyzed at $72 \mathrm{hr}$ post-transfection. (A) Transwell assay. Photographs represented the cells travelled through the micropore membrane and histogram showed the percentage of migrant cells. (B) Matrigel-coated transwell assay. Photographs represented the cells invaded through the matrigel and histogram showed the percentage of invasive cells. Bars $=100 \mu \mathrm{m}$. Data represent mean \pm SEM from 4 independent experiments; ${ }^{*}, P<0.05 ;{ }^{* *}, P<0.01$ by $t$ test.

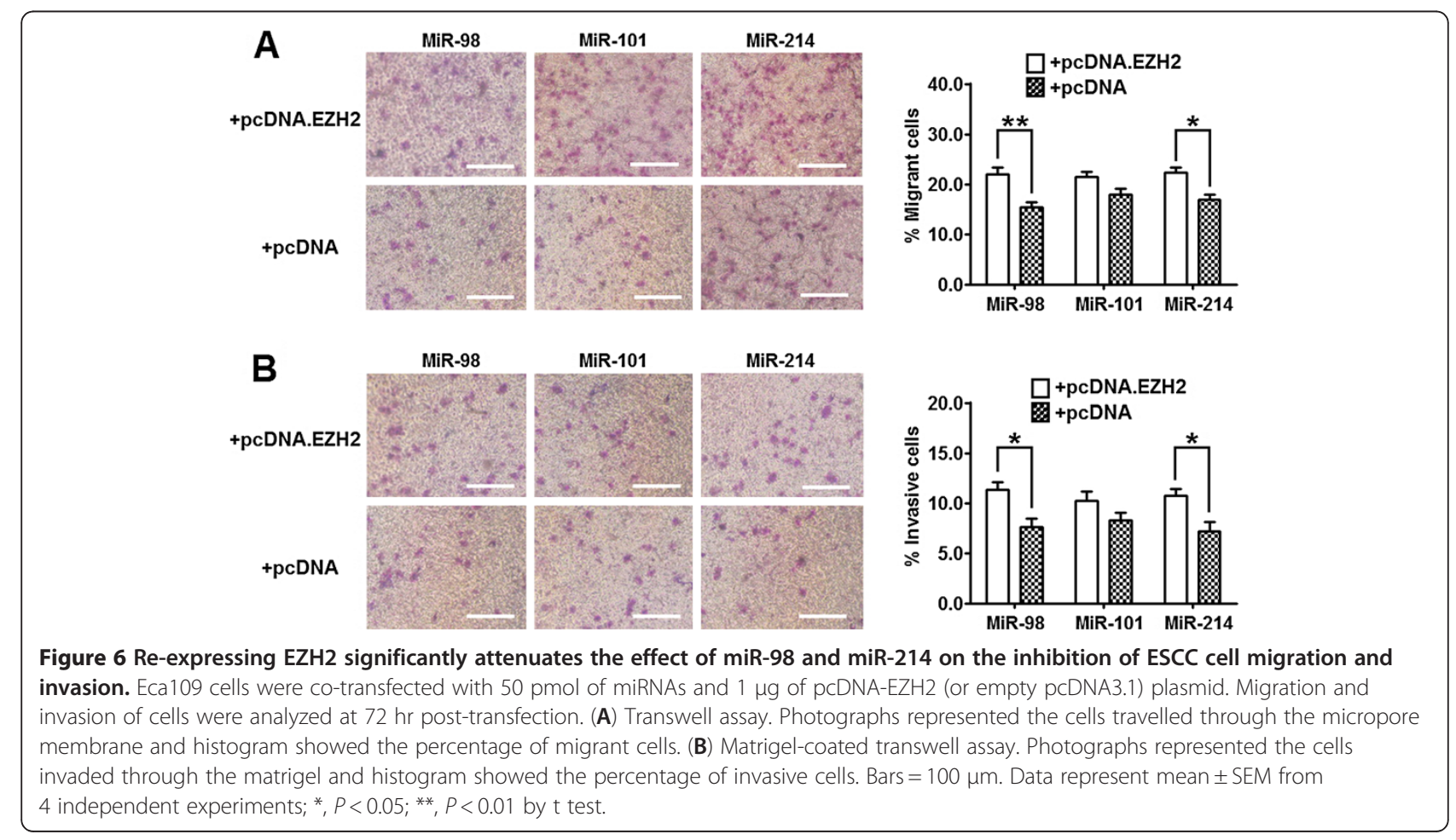


to carry the open reading frame of human $E Z H 2$ without 3'-UTR. As shown in Additional file 3: Figure S3, EZH2 protein expression was significantly higher in pcDNAEZH2 transfected cells than in pcDNA transfected cells. Compared with cells cotransfected with miR-98 (or miR-214) and empty cDNA plasmid, a significantly higher percentage of cells cotransfected with miR-98 (or miR-214) and pcDNA-EZH2 plasmid travelled through the micropore or invaded through the matrigel (Figure 6A and B). However, the percentage of cells that travelled through the micropore or invaded through the matrigel were comparable between cells cotransfected with miR-101 and pcDNA-EZH2 plasmid and cells cotransfected with miR101 and pcDNA plasmid (Figure 6A and B). These results show that overexpression of EZH2 could reverse the inhibitory effect of miR-98 and miR-214 on cell migration and invasion.

\section{Discussion}

EZH2 has been identified as a transcriptional repressor and is implicated in the aggressiveness and metastasis of many types of human cancers including ESCC $[19,20]$. In recent years, it was reported that some miRNAs could regulate EZH2 expression at the post-transcriptional level in several types of tumors [21-26]. In the present study, we show that miR-98 and miR-214 expression in ESCC tissue are inversely correlated with the clinical features such as pathological grade, tumor stage and lymph node metastasis. In Eca109 cells, overexpressing miR-98 and miR-214 was found to significantly suppress cell migration and invasion through inhibition of EZH2 expression.

Previous studies showed that the expression of EZH2 is regulated at the transcriptional and posttranscriptional levels [21-27]. Tang et al. [27] reported that p53 could inhibit the transcription of EZH2 by binding to the promoter of EZH2 in prostate cancer. On the other hand, mounting evidence indicate that some miRNAs could effectively repress the expression of EZH2 in tumors such as breast, prostate, endometrial, gastric, colon, hepatocellular, bladder and oral cancers [21-26]. In the present study, we detected EZH2 protein and mRNA expression in ESCC tumor tissues and matched normal tissues by western blot and qRT-PCR. It was found that the expression level of EZH2 protein was significantly higher in tumor tissues than in matched normal tissues, despite that EZH2 mRNA expression was comparable between the two groups. These results suggest that EZH2 expression is upregulated in human ESCC mainly at the posttranscriptional level.

MiRNAs are evolutionarily conserved small noncoding RNAs (21-25 nucleotides) that regulate gene expression through modulation of translation efficiency or degradation of mRNAs $[28,29]$. It was reported that miR-26a,
miR-98, miR-101, miR-124, miR-138 and miR-214 were involved in the regulation of EZH2 expression in some human tumors such as nasopharyngeal carcinoma, nasopharyngeal carcinoma, glioblastoma, hepatocellular carcinoma, head and neck squamous cell carcinoma, and neuroblastoma [21-26]. In the present study, we compared the expression level of these miRNAs in ESCC tissues and matched normal tissues by qPCR. We found that expression levels of miR-98, miR-101 and miR-214 were significantly lower in tumor than in normal tissues. On the other hand, miR-138 expression was significantly higher in tumor than in normal tissues and miR-26a and miR-124 expression was comparable between the two types of tissues. Using luciferase assay and western blot, we further demonstrated that miR-98, miR-101 and miR-214 could target the 3'-URT of EZH2 and suppress EZH2 expression in ESCC cells. Combining these findings, we propose that miR-98, miR-101 and miR-214 regulate the accumulation of EZH2 protein in ESCC.

MiR-98 belongs to the mature let-7 family of miRNAs [30] and was initially found to be down-regulated in leukemia cell lines [31]. Subsequent studies showed that the expression of miR-98 were also significantly decreased in solid tumors such as nasopharyngeal carcinoma, head and neck squamous cell carcinoma [22,32]. Therefore, miR-98 is wildly regarded as a tumor suppressor gene. On the other hand, the expression and function of miR-214 appears to be cell type- and disease-specific. It was reported that miR-214 was downregulated in breast and cervical cancer and acted as a tumor suppressor gene in these tumors since its overexpression inhibits cell proliferation and invasion [26,33]. By contrast, other studies showed that miR-214 was over-expressed in pancreatic and ovarian cancers and its overexpression promotes cell survival and chemotherapy resistance [34,35]. In the present study, we found that miR-98 and miR-214 expression were both inversely correlated with EZH2 protein expression in human ESCC, and that down-regulation of miR-98 and miR-214 expression was significantly correlated with pathological grading, tumor stage and lymph node metastasis. Moreover, overexpression of miR-98 or miR-214 could significantly inhibit ESCC cell migration and invasion, which was reversed by over-expressing EZH2. These findings suggest that miR-98 and miR-214 may play an important role in inhibiting the metastasis of ESCCs by targeting EZH2.

MiR-101 was reported to be down-regulated in human colon cancer, nasopharyngeal carcinoma, neuroblastoma and prostate cancer, and could repress the proliferation, invasion and metastasis of tumor cells [22,23,36,37]. In the present study, we found that miR-101 expression was down-regulated in primary ESCC tumor tissues and 
was significantly correlated with the tumor stage and lymph node metastasis. Although in Eca109 cells, over expression of miR-101 was found to suppress EZH2 expression, we did not detect significant correlation between the expression of miR-101 and EZH2 in clinical samples of ESSC tumor tissues and we found that miR101-induced inhibition of Eca109 migration and invasion was not reversed by overexpressing EZH2. The discrepancies might be attributable to the regulation of EZH2 expression by multiple miRNAs, amongst which miR101 only plays a minor role. Further studies are needed to test whether miR-101 might inhibit ESCC metastasis via an EZH2-independent signal pathway.

In summary, we have demonstrated that the expression of miR-98 and miR-214 was significantly lower in ESCC tissues than in matched normal tissues and that down-regulation of miR-98 and miR-214 was correlated with the up-regulated EZH2 protein expression, poor pathological grade, advanced tumor stage and lymph node metastasis in ESCC. In Eca109 cells, miR-98 and miR-214 overexpression significantly inhibited cell migration and invasion by repressing EZH2 protein expression. We propose that miR-98 and miR-214 are tumor suppressor genes in ESCC. It would be interesting to test whether these miRNAs act synergistically to regulate ESCC cell migration and invasion. Further experiments in animal models are warranted to establish the role of these miRNAs and EZH2 in regulating ESCC metastasis.

\section{Material and method ESCC specimens}

A total of forty primary ESCC patients that underwent esophagectomy were enrolled in this study. Tumor specimens and paired normal esophageal tissue specimens taken from a site distant from the cancerous lesion were obtained from the consenting patients, as approved by the Medical Ethics Committee of Changhai Hospital. None of the patients received radiotherapy or chemotherapy before surgery. Clinical and pathological data including age, gender, pathological grading, tumor location, tumor stage and lymph node metastasis were acquired from the medical records.

\section{Cell culture}

Human ESCC cell line Eca109 was purchased from the Shanghai Institute of Biochemistry and Cell Biology (Shanghai, China). Cells were maintained in RPMI1640 (Invitrogen) supplemented with 10\% fetal bovine serum (Invitrogen), $100 \mathrm{U} / \mathrm{ml}$ penicillin and $100 \mu \mathrm{g} / \mathrm{ml}$ streptomycin, within a humidified atmosphere containing 5\% $\mathrm{CO}_{2}$ at $37^{\circ} \mathrm{C}$.

\section{Cell transfection}

Cells were cultured to $1 \times 10^{6} /$ well in 6 -well cell culture plate and were then transfected with 50 pmol of miRNA double-stranded mimics (or control miRNA) using Lipofectamine 2000 (Invitrogen) according to the manufacturer's protocol Transfection efficiency was optimized using 6-carboxyfluorescein-labeled microRNA at approximately $80 \%$ in Eca109 cells.

The sequences of miR-98 were:

Sense: 5' - UGAGGUAGUAAGUUGUAUUGUU -3', Anti-sense: $5^{\prime}$ - AACAAUACAACUUACUACCUCA - $3^{\prime}$, The sequences of miR-101 were:

Sense: $5^{\prime}$ - UACAGUACUGUGAUAACUGAA - 3', Anti-sense: $5^{\prime}$ - UUCAGUUAUCACAGUACUGUA - $3^{\prime}$, The sequences of miR-214 were:

Sense: 5' - ACAGCAGGCACAGACAGGCAGU -3 , Anti-sense: $5^{\prime}$ - ACUGCCUGUCUGUGCCUGCUGU -3',

A scrambled microRNA with no homology to any known human microRNA was used as negative control:

Sense: $5^{\prime}$-GUUGAACUGUUAAGAACCACUGG-3', Anti-sense: 5'-CCAGUGGUUCUUAACAGUUCAAC-3', All microRNA mimics were synthesized by Genephama Biotech (Shanghai, China).

\section{Quantitative reverse transcription polymerase chain reaction (qRT-PCR)}

Total RNA was extracted from $100 \mathrm{mg}$ tissues or $1 \times 10^{5}$ cells using the RNeasy RNA Mini Kit (Qiagen). First strand cDNA was synthesized using POWERSCRIPT reverse transcriptase (Clontech). The following gene-specific primer pairs were used for quantitative PCR:

EZH2: Forward, 5' - TTACTTGTGGAGCCGCTGAC -3'; Reverse, $5^{\prime}$ - TCAGATGGTGCCAGCAATAG-3'.

GAPDH: Forward, 5'- GCTGAGTATGTCGTGGAGTC - $3^{\prime}$; Reverse, 5' - AGTTGGTGGTGCAGGATGC -3'.

PCR was performed using a Fast Start Master SYBR Green Kit (Roche) on a LightCycler (Roche). The expression level of target gene mRNA was analyzed using RealQuant software (Roche) and normalized to that of GAPDH mRNA.

\section{Cell lysis and western blot}

Cellular proteins were prepared using cell lysis buffer (50 mM Tris- $\mathrm{HCl}, \mathrm{pH}$ 8.0, 1\% NP-40, 2 mM EDTA, $10 \mathrm{mM} \mathrm{NaCl}, 2 \mathrm{mg} / \mathrm{ml}$ aprotinin, $5 \mathrm{mg} / \mathrm{ml}$ leupeptin, $2 \mathrm{mg} / \mathrm{ml}$ pepstatin, $1 \mathrm{mM}$ DTT, $0.1 \%$ SDS and $1 \mathrm{mM}$ phenylmethylsulfonyl fluoride). Equal amounts of protein $(50 \mu \mathrm{g})$ were separated by $10 \%$ SDS PAGE and then transferred to nitrocellulose membranes (NY, USA) by electroblotting. The membranes were blocked with $5 \%$ BSA in TBST (10 mM Tris-HCl, pH 8.0, $150 \mathrm{mM} \mathrm{NaCl}$, and $0.05 \%$ Tween 20) for $1 \mathrm{hr}$, and then incubated with mouse anti-human EZH2 antibody (Santa Cruz) 
overnight at $4^{\circ} \mathrm{C}$ before subsequent incubation with horseradish peroxidase-conjugated goat anti-mouse antibody (BD) for $1 \mathrm{hr}$ at $37^{\circ} \mathrm{C}$. Protein was visualized using enhanced chemiluminescence reagent (Santa Cruz). The expression level of EZH2 protein was analyzed using LabWork 4.0 program (UVP) and normalized to that of $\beta$-actin protein.

\section{Luciferase reporter assay}

The full-length $3^{\prime}$-UTR of EZH2 mRNA was amplified by PCR (Forward: 5'-CTAGTCATCTGCTACCTCCTCC-3'; Reverse: 5'-AGCTTACAAGTTCAAGTATTCTTTATTC3'). Mutant EZH2 3'-UTR, which carried a substitution of four nucleotides (AGGU to UCCA for miR-98, UACU to AUGA for miR-101, and CAGC to GUCG for miR214) within the core binding sites of EZH2 $3^{\prime}$-UTR, was obtained using overlapping extension PCR. Normal (or mutant) EZH2 3'-UTR was cloned into the SacI-HindIII site of the pMIR-REPORT luciferase vector (Biosystems) and named as Luc-EZH2 (or Luc-EZH2-mut). Then, $1 \times 10^{6}$ cells were cotransfected with 50 pmol of miRNAs (or control miRNA), $1 \mu \mathrm{g}$ of Luc-EZH2 (or matched Luc-EZH2-mut) plasmid, and $1 \mu \mathrm{g}$ of pMIR-REPORT $\beta$-Gal vector using Lipofectamine 2000. The Luciferase activity was examined at $48 \mathrm{hr}$ posttransfection using the luciferase assay kit (Clontech) and normalized to $\beta$-galactosidase activity.

\section{Transwell assay}

Cell migration and invasion were determined using a transwell (Costar) with a pore size of $0.8 \mu \mathrm{m} .1 \times 10^{3}$ cells were seeded in serum-free medium in the upper chamber (normal chamber for migration assay and matrigel-coated chamber for invasion assay). The lower chamber was filled with medium containing $10 \%$ FBS. After incubating for $8 \mathrm{hr}$ at $37^{\circ} \mathrm{C}$, cells in the upper chamber were carefully removed with a cotton swab and the cells that had traversed to reverse face of the membrane were fixed in methanol, stained with Giemsa, and counted.

\section{Statistical analysis}

Statistical significance was tested using SPSS15.0 software. For comparison of clinical features (except for pathological grading) between high and low miRNA expression groups, chi-square test was performed. The correlation between the expression of miRNA and pathological grade was analyzed by Cochran-MantelHaenszel Statistics. The correlation between the expression of miRNA and EZH2 was analyzed using Pearson's correlation analysis. Other data are presented as mean $\pm \mathrm{SEM}$, using student $t$ tests for 2-group comparison. A $P$ value less than 0.05 is considered as statistically significant.

\section{Additional files}

Additional file 1: Figure S1. The expression level of EZH2 protein in ESCC and paired normal tissues.

Additional file 2: Figure S2. The expression levels of miRNAs were significantly increased in Eca109 cells transfected with miRNA mimics.

Additional file 3: Figure S3. The expression levels of EZH2 was significantly increased in Eca109 cells transfected with pcDNA.EZH2.

\section{Abbreviations}

miR: microRNA; ESCC: Esophageal squamous cell carcinoma; EZH2: Enhancer of zeste homolog 2; 3'-UTR: 3' untranslated region.

\section{Competing interests}

The authors declare that they have no competing interests.

\section{Authors' contributions}

SDH and ZYZ designed research and analyzed data. YY, HZC, BLL, DJG, CWZ and SGW carried out molecular biology studies. YY and HZC wrote the paper. All authors read and approved the final manuscript.

\section{Acknowledgments}

This work was supported by the National Natural Science Foundation of China (grants 30872552 and 81272666), the Shanghai Municipal Natural Science Foundation (grant 10140902300).

\section{Author details}

${ }^{1}$ Institute of Cardiothoracic Surgery, Changhai Hospital, 168, Changhai Rd., Shanghai, P. R. China. ${ }^{2}$ Department of Cardiothoracic Surgery, Changhai Hospital, Shanghai, P.R. China. ${ }^{3}$ Department of Pathology, Changhai Hospital, Second Military Medical University, Shanghai, P.R. China. ${ }^{4}$ Department of Cardiothoracic Surgery, Fuzhou General Hospital of Nanjing Command, PLA., Fujian, P.R. China. ${ }^{5}$ Department of Pathology, Fuzhou General Hospital of Nanjing Command, PLA, Fujian, P.R. China.

Received: 5 May 2012 Accepted: 26 July 2012

Published: 6 August 2012

\section{References}

1. Enzinger PC, Mayer RJ: Esophageal cancer. N Engl J Med 2003, 349(23):2241-2252.

2. Gamliel Z, Krasna MJ: Multimodality treatment of esophageal cancer. Surg Clin North Am 2005, 85(3):621-630.

3. Feber A, Xi L, Luketich JD, Pennathur A, Landreneau RJ, Wu M, Swanson SJ, Godfrey TE, Litle VR: MicroRNA expression profiles of esophageal cancer. J Thorac Cardiovasc Surg 2008, 135(2):255-260. discussion 260.

4. Ren Y, Cao B, Law S, Xie Y, Lee PY, Cheung L, Chen Y, Huang X, Chan HM, Zhao P, Luk J: Vande Woude G, Wong J: Hepatocyte growth factor promotes cancer cell migration and angiogenic factors expression: a prognostic marker of human esophageal squamous cell carcinomas. Clin Cancer Res 2005, 11(17):6190-6197.

5. Xie F, Li K, Ouyang X: Twist, an independent prognostic marker for predicting distant metastasis and survival rates of esophageal squamous cell carcinoma patients. Clinical \& experimental metastasis 2009, 26(8):1025-1032

6. Wang X, Tian X, Liu F, Zhao Y, Sun M, Chen D, Lu C, Wang Z, Shi X, Zhang Q, Zhang D, Shen Z, Li F, Harris CC, Cai H, Ke Y: Detection of HPV DNA in esophageal cancer specimens from different regions and ethnic groups: a descriptive study. BMC Cancer 2010, 10:19.

7. Sudo T, Utsunomiya T, Mimori K, Nagahara H, Ogawa K, Inoue H, Wakiyama S, Fujita H, Shirouzu K, Mori M: Clinicopathological significance of EZH2 mRNA expression in patients with hepatocellular carcinoma. $\mathrm{Br} J$ Cancer 2005, 92(9):1754-1758.

8. Saramaki OR, Tammela TL, Martikainen PM, Vessella RL, Visakorpi T: The gene for polycomb group protein enhancer of zeste homolog 2 (EZH2) 
is amplified in late-stage prostate cancer. Genes Chromosomes Cancer 2006, 45(7):639-645.

9. Tsang DP, Cheng AS: Epigenetic regulation of signaling pathways in cancer: role of the histone methyltransferase EZH2. J Gastroenterol Hepatol 2011, 26(1):19-27.

10. Chang CJ, Hung MC: The role of EZH2 in tumour progression. Br J Cancer 2012, 106(2):243-247.

11. Kleer CG, Cao Q, Varambally S, Shen R, Ota I, Tomlins SA, Ghosh D, Sewalt RG, Otte AP, Hayes DF, Sabel MS, Livant D, Weiss SJ, Rubin MA, Chinnaiyan AM: EZH2 is a marker of aggressive breast cancer and promotes neoplastic transformation of breast epithelial cells. Proc Natl Acad Sci U S A 2003, 100(20):11606-11611.

12. van Leenders GJ, Dukers D, Hessels D, van den Kieboom SW, Hulsbergen CA, Witjes JA, Otte AP, Meijer CJ, Raaphorst FM: Polycomb-group oncogenes EZH2, BMI1, and RING1 are overexpressed in prostate cancer with adverse pathologic and clinical features. Eur Urol 2007, 52(2):455-463.

13. Bachmann IM, Halvorsen OJ, Collett K, Stefansson IM, Straume O, Haukaas SA, Salvesen HB, Otte AP, Akslen LA: EZH2 expression is associated with high proliferation rate and aggressive tumor subgroups in cutaneous melanoma and cancers of the endometrium, prostate, and breast. J Clin Oncol 2006, 24(2):268-273.

14. Mattioli E, Vogiatzi P, Sun A, Abbadessa G, Angeloni G, D'Ugo D, Trani D, Gaughan JP, Vecchio FM, Cevenini G, Persiani R, Giordano A, Claudio PP: Immunohistochemical analysis of pRb2/p130, VEGF, EZH2, p53, p16 (INK4A), p27(KIP1), p21(WAF1), Ki-67 expression patterns in gastric cancer. J Cell Physiol 2007, 210(1):183-191.

15. Crea F, Fornaro L, Paolicchi E, Masi G, Frumento P, Loupakis F, Salvatore L, Cremolini C, Schirripa M, Graziano F, Ronzoni M, Ricci V, Farrar WL, Falcone A, Danesi R: An EZH2 polymorphism is associated with clinical outcome in metastatic colorectal cancer patients. Ann Oncol 2012, 23(5):1207-1213.

16. Chen Y, Lin MC, Wang H, Chan CY, Jiang L, Ngai SM, Yu J, He ML, Shaw PC, Yew DT, Sung JJ, Kung HF: Proteomic analysis of EZH2 downstream target proteins in hepatocellular carcinoma. Proteomics 2007, 7(17):3097-3104.

17. Raman JD, Mongan NP, Tickoo SK, Boorijan SA, Scherr DS, Gudas LJ: Increased expression of the polycomb group gene, EZH2, in transitional cell carcinoma of the bladder. Clin Cancer Res 2005, 11(24 Pt 1):8570-8576.

18. Kidani K, Osaki M, Tamura T, Yamaga K, Shomori K, Ryoke K, Ito H: High expression of $\mathrm{EZH} 2$ is associated with tumor proliferation and prognosis in human oral squamous cell carcinomas. Oral oncology 2009, 45(1):39-46.

19. He LR, Liu MZ, Li BK, Jia WH, Zhang Y, Liao YJ, Chen YC, Zhang LJ, Guan XY, Zeng $Y X$, Kung HF, Xie D: High expression of EZH2 is associated with tumor aggressiveness and poor prognosis in patients with esophageal squamous cell carcinoma treated with definitive chemoradiotherapy. Int J Cancer 2010, 127(1):138-147.

20. Tzao C, Tung HJ, Jin JS, Sun GH, Hsu HS, Chen BH, Yu CP, Lee SC: Prognostic significance of global histone modifications in resected squamous cell carcinoma of the esophagus. Mod Pathol 2009, 22(2):252-260.

21. Lu J, He ML, Wang L, Chen Y, Liu X, Dong Q, Chen YC, Peng Y, Yao KT, Kung HF, Li XP: MiR-26a inhibits cell growth and tumorigenesis of nasopharyngeal carcinoma through repression of EZH2. Cancer Res 2011, 71(1):225-233.

22. Alajez NM, Shi W, Hui AB, Bruce J, Lenarduzzi M, Ito E, Yue S, O'Sullivan B, Liu FF: Enhancer of Zeste homolog $2(\mathrm{EZH} 2)$ is overexpressed in recurrent nasopharyngeal carcinoma and is regulated by miR-26a, miR101, and miR-98. Cell death \& disease 2010, 1:e85.

23. Cao P, Deng Z, Wan M, Huang W, Cramer SD, Xu J, Lei M, Sui G: MicroRNA-101 negatively regulates Ezh2 and its expression is modulated by androgen receptor and HIF-1alpha/HIF-1 beta. Mol Cancer 2010, 9:108.

24. Zheng F, Liao YJ, Cai MY, Liu YH, Liu TH, Chen SP, Bian XW, Guan XY, Lin $M C$, Zeng $Y X$, Kung HF, Xie D: The putative tumour suppressor microRNA124 modulates hepatocellular carcinoma cell aggressiveness by repressing ROCK2 and EZH2. Gut 2012, 61(2):278-289.

25. Kisliouk T, Yosefi S, Meiri N: MiR-138 inhibits EZH2 methyltransferase expression and methylation of histone $\mathrm{H} 3$ at lysine 27 , and affects thermotolerance acquisition. Eur J Neurosci 2011, 33(2):224-235.

26. Derfoul A, Juan AH, Difilippantonio MJ, Palanisamy N, Ried T, Sartorelli V: Decreased microRNA-214 levels in breast cancer cells coincides with increased cell proliferation, invasion and accumulation of the Polycomb Ezh2 methyltransferase. Carcinogenesis 2011, 32(11):1607-1614.

27. Tang X, Milyavsky M, Shats I, Erez N, Goldfinger N, Rotter V: Activated p53 suppresses the histone methyltransferase EZH2 gene. Oncogene 2004, 23(34):5759-5769.

28. Esquela-Kerscher A, Slack FJ: Oncomirs - microRNAs with a role in cancer. Nat Rev Cancer 2006, 6(4):259-269.

29. Chen K, Rajewsky N: The evolution of gene regulation by transcription factors and microRNAs. Nat Rev Genet 2007, 8(2):93-103.

30. Roush S, Slack FJ: The let-7 family of microRNAs. Trends Cell Biol 2008, 18(10):505-516.

31. Yu J, Wang F, Yang GH, Wang FL, Ma YN, Du ZW, Zhang JW: Human microRNA clusters: genomic organization and expression profile in leukemia cell lines. Biochem Biophys Res Commun 2006, 349(1):59-68.

32. Hebert C, Norris K, Scheper MA, Nikitakis N, Sauk JJ: High mobility group A2 is a target for miRNA-98 in head and neck squamous cell carcinoma. Mol Cancer 2007, 6:5.

33. Yang Z, Chen S, Luan X, Li Y, Liu M, Li X, Liu T, Tang H: MicroRNA-214 is aberrantly expressed in cervical cancers and inhibits the growth of HeLa cells. IUBMB life 2009, 61(11):1075-1082.

34. Zhang XJ, Ye H, Zeng CW, He B, Zhang H, Chen YQ: Dysregulation of miR-15a and miR-214 in human pancreatic cancer. Journal of hematology \& oncology 2010, 3:46.

35. Yang H, Kong W, He L, Zhao JJ, O'Donnell JD, Wang J, Wenham RM, Coppola D, Kruk PA, Nicosia SV, Cheng JQ: MicroRNA expression profiling in human ovarian cancer: miR-214 induces cell survival and cisplatin resistance by targeting PTEN. Cancer Res 2008, 68(2):425-433.

36. Buechner J, Tomte E, Haug BH, Henriksen JR, Lokke C, Flaegstad T, Einvik C: Tumour-suppressor microRNAs let-7 and mir-101 target the protooncogene MYCN and inhibit cell proliferation in MYCN-amplified neuroblastoma. Br J Cancer 2011, 105(2):296-303.

37. Strillacci A, Griffoni C, Sansone P, Paterini P, Piazzi G, Lazzarini G, Spisni E, Pantaleo MA, Biasco G, Tomasi V: MiR-101 downregulation is involved in cyclooxygenase-2 overexpression in human colon cancer cells. Exp Cell Res 2009, 315(8):1439-1447.

doi:10.1186/1476-4598-11-51

Cite this article as: Huang et al:: MicroRNA-98 and microRNA-214

post-transcriptionally regulate enhancer of zeste homolog 2 and inhibit migration and invasion in human esophageal squamous cell carcinoma. Molecular Cancer 2012 11:51.

\section{Submit your next manuscript to BioMed Central and take full advantage of:}

- Convenient online submission

- Thorough peer review

- No space constraints or color figure charges

- Immediate publication on acceptance

- Inclusion in PubMed, CAS, Scopus and Google Scholar

- Research which is freely available for redistribution
C Biomed Central 